\title{
Synthesis of 2, 6-Diaryl-4-5-Secondary aminonicotinonitriles as potent antimicrobial agents
}

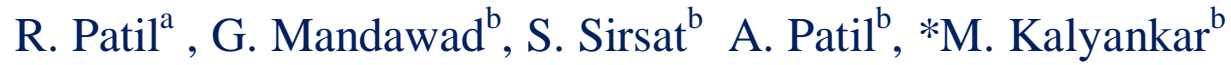 \\ ${ }^{a}$ Department of Botany, Yeshwant Mahavidyalaya, Nanded (India) \\ ${ }^{b}$ Department of Chemistry, Yeshwant Mahavidyalaya, Nanded (India)
}

\begin{abstract}
A series of 2, 6-Diaryl-4-5-Secondary aminonicotinonitriles derivatives were synthesized by the reaction of 2Hpyran-2-one (I) and the $\mathrm{N}$-aryl amidine (II) using $\mathrm{KOH}$ as catalyst in DMF at room temperature. All synthesized compounds (IIIa-i) evaluated for antibacterial activity against Staphylococcus aureus, Bacillus subtilis, Escherichia coli, Proteus vulgaris, Aspergillus niger and, Candida albicans strains most of the compound shows potent activity.
\end{abstract}

Keywords: 2-H-pyran-2-one, N-aryl amidine, aminonicotinonitrile and Antimicrobial activity.

\section{Introduction}

Pyridine and its derivatives are one of the most important class of heterocycles found in many natural products like vitamin-B12 (Niacin and Pyridoxal), alkaloids (Nicotine) and it act as key precursor for synthesis of various medicines and agrochemicals. In recent past, after considering the novel insecticides belonging to the group, neonicotinoids[1-2] like imidacloprid and nicotin novel derivatives of pyridine have been developed and used as antibacterial, antifungal[3], insecticidal[4] and pesticidal[5-6] agents. It was also found that 2-(p-aminobenzamido) pyridines exhibit a powerful inhibiting effect on gastric ulcers in rats [7]. The pyridines 2,2'-bi- and 2,2', 2' '-ter-pyridine were used as metal chelating legends with various substituents [8]. Similarly, pyridine derivatives are of growing relevance in material science and supramolecular chemistry [9]. Therefore, there is a continuous interest to develop the new synthetic methods for pyridines and their derivatives. Classical routes to pyridine synthesis discovered by Hantzsch [10], Chichibabin [11], PetrenkoKritschenko and Zoneff [12], Krohnke [13], and Guareschi-Thorpe [14] condensation reactions. The condensation of 1,5diketone with ammonia followed by nitric acid oxidation is a common approach for the synthesis of pyridines [15]. The reaction of dienamine and ketone in the presence of Vilsmeier type 1-substituted-1,2,3-benzotriazole reagent results in the formation of nicotinonitriles [16]. The construction of unsymmetrically substituted pyridines was achieved by the reaction of 1,3-dicarbonyl compounds and 3-aminoenones or nitriles [17]. Saikai et al. reported indium trichloride catalyzed synthesis of tetrasubstituted pyridines [18]. Penieres et al. have synthesized pyridine by using microwave irradiated Hantzsch reaction [19]. Combinatorial approach also has been used for the synthesis of pyridine derivatives [20]. 2,4,6-trisubstituted pyridine derivatives were prepared from aroylketene dithioacetal by Potts et al [21]. Recently, Ram and coworkers [22] have described the use of 2H-pyran-2-one for the synthesis of substituted pyridines. The chemical structures of the synthesized compounds were confirmed by means of IR, 1H-NMR, Mass and C, H, N elemental analysis. The synthesized compounds were screened for antibacterial activity against Staphylococcus aureus, Escherichia coli, Bacillus subtilis, Pseudomonas aeruginosa and fungi Aspergillus niger and Candida albicans.

\section{Results and Discussion}

2.1 Chemistry

We investigated that compound III a-i in moderate yield can be constructed from 2H-pyran-2-one I and the N-aryl amidine II via a ring transformation reaction using $\mathrm{KOH}$ in DMF at room temperature (Scheme1). Pyridine III a-i isolated in this study could arise by nucleophilic attack of amidine N-1 at C-6 position of $2 \mathrm{H}$-pyran-2-one. The intermediate 4 formed is unstable and it undergoes cyclization with a retro [2+2] process to yield III a-i with the loss of carbon dioxide and yields 2,6-Diaryl-4-5-Secondary aminonicotinonitriles as target compounds.

\subsection{Microbiology}

The antimicrobial activities of these compounds were evaluated by minimum inhibitory concentration (MIC) against Gram-positive and Gram-negative test bacteria Staphylococcus aureus (MTCC 96), Bacillus subtilis (MTCC 441), Escherichia coli (MTCC 1650), Pseudomonas aeruginosa, (MTCC 1688), and fungi Aspergillus niger (MTCC 1789) and Candida albicans (MTCC 227) in vitro. Streptomycin and nystatin as reference drug, by agar diffusion method, [23-26] the 
results of in vitro antibacterial activities and MIC's of compound III a-i against various bacterial strains are summarized in Table 2, compound III a, III c, shows excellent activity against all bacteria with showing more than $12 \mathrm{~mm}$ zone of inhibition at $25 \mu \mathrm{g} / \mathrm{mL}$ MIC value. Compound III f is found to be potent activity against Bacillus subtilis and Escherichia coli even at very low concentration where as compound III i also point out excellent activity against Pseudomonas aeruginosa, with showing above $15 \mathrm{~mm}$ zone of inhibition even at 25 MIC value in competition with standard drug, where as compound III $\mathbf{f}$ is found to be almost equally potent active against Escherichia coli in comparison with streptomycin even at lowest MIC (25 ug /ml). Compound III b, III d, III e, and III h are also found good active but at double concentration. Compound III a shows considerable activity against Aspergillus niger where as compound III c and III f shows good activity against both fungal species. Compound III i found to most potent active towards Aspergillus niger and Candida albicans with showing more than $12 \mathrm{~mm}$ zone of inhibition at $25 \mu \mathrm{g} / \mathrm{mL}$ MIC value in comparison with standard drug. Likewise remaining compound III b, III d, III e, III $\mathbf{g}$ and, III $\mathbf{h}$ are also found active but above $25 \mu \mathrm{g} / \mathrm{mL}$.

\section{Experimental}

\subsection{Chemistry}

All chemicals and solvents used were laboratory grade and directly used. Melting points were determined by open capillary method and are uncorrected. ${ }^{1} \mathrm{H}$ NMR spectra were recorded (in DMSO- $d_{6}, \square$ ppm) on AVANCE-300 MHz spectrometer using TMS as an internal standard ( $\mathrm{s}=$ singlet, $\mathrm{d}=$ doublet, $\mathrm{t}=$ triplet, $\mathrm{m}=$ multiplates and br $=$ brod). Coupling constant $(J)$ are given in $(\mathrm{Hz})$. IR spectra were recorded (in $\mathrm{KBr}$ pallets) on SCHIMADZU spectrophotometer. Mass spectra were recorded on EI-SHIMDZU-GC-MS spectrometer. Elemental analyses were performed on a Perkins-Elmer C, H, N, elemental analyzer. All reactions and purity of isolated product was monitored by using thin layer chromatography (TLC) using $0.2 \mathrm{~mm}$ silica gel plates $60 \mathrm{~F}_{254}$ (MERCK) and mobile phase petroleum ether and ethyl acetate (80:20). Reaction components were visualized in UV (255 and $365 \mathrm{~nm})$ and iodine chamber.

\subsection{General procedure for Preparation of 2,6-Diaryl-4-secondary aminonicotinonitriles (III a-i)}

The mixture of 2H-pyran-2-one-3-carbonitrile $(1.0 \mathrm{mmol}), \mathrm{N}$-phenyl benzamidine $(1.0 \mathrm{mmol})$ and powdered $\mathrm{KOH}(2.0$ $\mathrm{mmol}$ ) in $5 \mathrm{~mL}$ DMF was stirred for $5-6 \mathrm{~h}$ at room temperature. The reaction was monitored by TLC. After completion of the reaction, excess DMF was removed under reduced pressure. Then the residue was poured into crushed ice with vigorous stirring. The aqueous solution was neutralized with $1 \mathrm{~N}, \mathrm{HCl}$, the precipitate obtained was filtered, and the obtained residue was purified by column chromatography on silica gel 60-120 by eluting 5:95 ethyl acetate: hexane as mobile phase.

\section{Spectral data of selected compounds}

IIIa) 2,6-Diphenyl-4-(piperidin-1-yl)pyridine-3-carbonitrile : White solid, IR (KBr): $2209(\mathrm{CN}), 1568(\mathrm{CN}) \mathrm{cm}^{-1} .1 \mathrm{H}$ NMR (300 MHz, $\left.\mathrm{CDCl}_{3}, 25^{\circ} \mathrm{C}\right) \square 1.73\left(\mathrm{~d}, \mathrm{~J}=5.7 \mathrm{~Hz}, 2 \mathrm{H}, \mathrm{CH}_{2}\right), 1.83\left(\mathrm{~d}, \mathrm{~J}=3.3 \mathrm{~Hz}, 4 \mathrm{H}, 2 \mathrm{CH}_{2}\right), 3.54(\mathrm{t}, \mathrm{J}=9.9 \mathrm{~Hz}, 4 \mathrm{H}$, $\left.2 \mathrm{CH}_{2} \mathrm{~N}\right), 6.91(\mathrm{~s}, 1 \mathrm{H}, \mathrm{CH}), 7.5(\mathrm{~m}, 5 \mathrm{H}, \mathrm{ArH}), 7.84(\mathrm{~d}, \mathrm{~J}=4.7 \mathrm{~Hz}, 2 \mathrm{H}, \mathrm{ArH}), 8.05(\mathrm{t}, \mathrm{J}=9.5 \mathrm{~Hz}, 3 \mathrm{H}, \mathrm{ArH}) .{ }^{13} \mathrm{C} \mathrm{NMR}(75$ $\left.\mathrm{MHz}, \mathrm{CDCl}_{3}\right) \square=24.2,26.1,52.1,96.4,106.7,118.7,124.4,125.3,127.6,128.5,128.9,129.6,130.1,138.9,159.8,162.9$, 164.2. MS (ESI, $70 \mathrm{eV}) \mathrm{m} / \mathrm{z}(\%)=340(100)[\mathrm{M}+], 341(27)\left[(\mathrm{M}+\mathrm{H})^{+}\right]$. Anal. Calcd. for $\mathrm{C}_{23} \mathrm{H}_{21} \mathrm{~N}_{3}: \mathrm{C}, 81.38 ; \mathrm{H}, 6.24 ; \mathrm{N}$, 12.38. Found: C, 81.42; H, 6.20; N, 12.38 .

IIIc) 6-(4-Bromophenyl)-2-phenyl-4-(piperidin-1-yl)pyridine-3- carbonitrile: White solid, IR (KBr): 2212 (CN), 1568 $(\mathrm{CN}) \mathrm{cm}^{-1}$. 1H NMR (300 MHz, $\left.\mathrm{CDCl}_{3}, 25^{\circ} \mathrm{C}\right) \square=1.72\left(\mathrm{~d}, 2 \mathrm{H}, \mathrm{CH}_{2}\right), 1.8\left(\mathrm{~d}, \mathrm{~J}=5 \mathrm{~Hz}, 4 \mathrm{H}, 2 \mathrm{CH}_{2}\right), 3.54(\mathrm{t}, \mathrm{J}=10.6 \mathrm{~Hz}, 4 \mathrm{H}$, $\left.2 \mathrm{CH}_{2} \mathrm{~N}\right), 7.12(\mathrm{~s}, 1 \mathrm{H}, \mathrm{CH}), 7.51(\mathrm{~m}, 3 \mathrm{H}, \mathrm{ArH}), 7.59(\mathrm{~s}, 1 \mathrm{H}, \mathrm{ArH}), 7.62(\mathrm{~s}, 1 \mathrm{H}, \mathrm{ArH}), 7.93(\mathrm{~m}, 4 \mathrm{H}, \mathrm{ArH}) .13 \mathrm{C} \mathrm{NMR}(75 \mathrm{MHz}$, $\mathrm{CDCl} 3) \square=24.2,26.1,52.0,96.8,106.4,118.6,124.7,128.6$ 129.2, 129.6, 130.1, 132.1, 137.7, 138.7, 158.5, 162.9, 163.9. MS (ESI, $70 \mathrm{eV}) \mathrm{m} / \mathrm{z}(\%)=418(100)[\mathrm{M}+], 420(92)[(\mathrm{M}+2 \mathrm{H})+]$. Anal. Calcd. for $\mathrm{C}_{23} \mathrm{H}_{20} \mathrm{BrN}_{3}$ : C, 66.04; H, 4.82; N, 10.04. Found: C, 66.01; H, 4.77; N, 10.08 .

IIIf) 6-(4-Chlorophenyl)-4-(morpholin-4-yl)-2-phenylpyridine-3-carbonitrile: White solid, IR (KBr): $2216(\mathrm{CN}), 1583$ $(\mathrm{C}=\mathrm{N}) \mathrm{cm}^{-1}$. 1H NMR $\left(300 \mathrm{MHz}, 3,25^{\circ} \mathrm{C}\right) \mathrm{d}=3.54\left(\mathrm{q}, \mathrm{J}=14.1 \mathrm{~Hz}, 4 \mathrm{H}, 2 \mathrm{CH}_{2} \mathrm{~N}\right), 3.94\left(\mathrm{q}, \mathrm{J}=13.9 \mathrm{~Hz}, 4 \mathrm{H}, 2 \mathrm{CH}{ }_{2} \mathrm{O}\right), 7.14(\mathrm{~s}$, $\left.1 \mathrm{H}, \mathrm{CH}), 7.51(\mathrm{~m}, 5 \mathrm{H}, \mathrm{ArH}), 7.89(\mathrm{~m}, 2 \mathrm{H}, \mathrm{ArH}), 8.00(\mathrm{~s}, 1 \mathrm{H}, \mathrm{ArH}), 8.04(\mathrm{~s}, 1 \mathrm{H}, \mathrm{ArH}) .13 \mathrm{C} \mathrm{NMR}\left(75 \mathrm{MHz}^{\mathrm{CDCl}}\right)_{3}\right) \mathrm{d}=50.9$, 66.8, 97.2, 106.3, 118.2, 128.6, 128.9, 129.3, 129.5, 130.3, 136.7, 136.9, 138.3, 159.0, 162.7, 164.2. MS (ESI, 70 eV) m/z (\%): $376(100)[\mathrm{M}+], 378(33)[(\mathrm{M}+2 \mathrm{H})+]$. Anal. Calcd. for $\mathrm{C}_{22} \mathrm{H}_{18} \mathrm{ClN}_{3} \mathrm{O}: \mathrm{C}, 70.30 ; \mathrm{H}, 4.83 ; \mathrm{N}, 11.18$. Found: C, 70.34; H, 4.94; N, 11.23.

IIIh) 6-(4-Chlorophenyl)-2-phenyl-4-(pyrrolidin-1-yl)pyridine-3-carbonitrile: White solid, IR (KBr): 2199 (CN), 1590 $(\mathrm{C}=\mathrm{N}) \mathrm{cm}^{-1}$. 1H NMR $\left(300 \mathrm{MHz}, \mathrm{CDCl} 3,25^{\circ} \mathrm{C}\right) \mathrm{d} 2.09\left(\mathrm{~m}, 4 \mathrm{H}, 2 \mathrm{CH}_{2}\right), 3.81\left(\mathrm{t}, \mathrm{J}=13.2 \mathrm{~Hz}, 4 \mathrm{H}, 2 \mathrm{CH}{ }_{2} \mathrm{~N}\right), 6.85(\mathrm{~s}, 1 \mathrm{H}, \mathrm{CH})$, 
7.48 (m, 5H, ArH), 7.86 (q, J = 9.6 Hz, 2H, ArH), 7.98 (d, J = 8.66 Hz, 2H, ArH). 13C NMR (75 MHz, CDCl $)^{\prime} \square=25.9$, $50.5,96.1,106.5,118.6,128.4,128.6,129.0,129.6,129.8,135.9,137.5,139.3,158.6,162.8,163.1 . \mathrm{MS}(\mathrm{ESI}, 70 \mathrm{eV}) \mathrm{m} / \mathrm{z}$ $(\%)=360(100)[\mathrm{M}+], 362(32)\left[(\mathrm{M}+2 \mathrm{H})^{+}\right]$. Anal. Calcd. for $\mathrm{C}_{22} \mathrm{H}_{18} \mathrm{ClN}_{3}: \mathrm{C}, 73.43 ; \mathrm{H}, 5.04 ; \mathrm{N}, 11.68$. Found: C,73.42; H, 5.04; N, 11.03 .

\subsection{Determination of Antimicrobial Activity \\ 3.3.1. Antibacterial activity}

The antibacterial activities of the synthesized compounds III a-i were determined by agar diffusion method as recommended by the National Committee for Clinical Laboratory Standards, (NCCLS) [23-25], against selected Grampositive bacteria viz. Bacillus subtilis (MTCC 441) and Staphylococcus aureus (MTCC 96) and Gram-negative bacteria viz. Pseudomonas aeruginosa (MTCC 1688), and Escherichia coli (MTCC 1650) strains by the agar well diffusion method. Briefly, $0.1 \mathrm{~mL}$ of overnight grown respective bacterial culture was spreaded over the nutrient agar plates. The wells of $6 \mathrm{~mm}$ diameter were prepared on the nutrient agar plates and filled with diluted test compounds separately. For comparison, DMSO and antibiotic Streptomycin were used as a solvent control and as reference antibacterial agent, respectively. Inoculated plates were then incubated at $37^{\circ} \mathrm{C}$ for $24 \mathrm{~h}$ and the resulting zones of inhibition (in mm) were measured. The minimum inhibitory concentrations at which no growth was observed was taken as the MIC value.

\subsubsection{Antifungal activity}

The compounds were screened for their antifungal activity on the fungal strains Aspergillus niger (MTCC 1789) and Candida albicans (MTCC 227). Fungal suspension (0.1 mL) was spread on Sabourauds agar plates. The wells of $6 \mathrm{~mm}$ diameter were prepared on the inoculated plates and filled with diluted test compounds separately. For comparison, DMSO and antibiotic Nystatin were used as solvent control and reference antifungal agent, respectively. Inoculated plates were then incubated at $30^{\circ} \mathrm{C}$ for $2-3$ days and the resulting zones of inhibition (in $\mathrm{mm}$ ) were measured. The minimum inhibitory concentrations at which no fungal growth observed was recorded as the MIC value.

\section{Figuers and Tables}

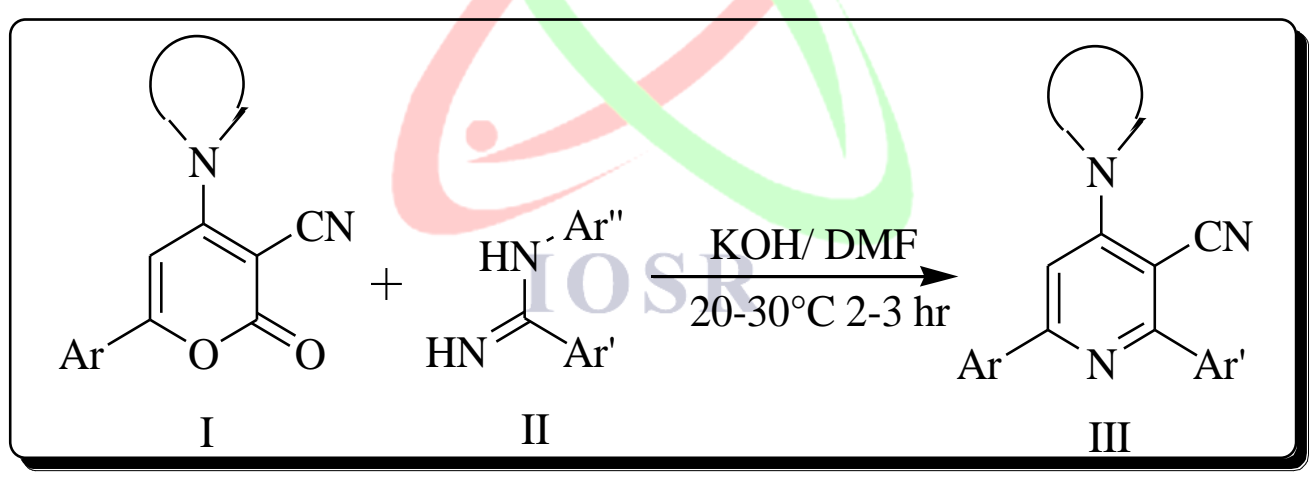

Sheme-1 
Table: 1 Preparation of 2, 6-Diaryl-4-secondary aminonicotinonitriles 3 from 2H-pyran-2-one 1 and $\mathrm{N}$-aryl amidine

\begin{tabular}{|c|c|c|c|c|c|c|}
\hline & & & & & & \\
\hline Entry & $\mathrm{Ar}$ & $\mathrm{HN}^{\prime}$ & $\mathrm{Ar}^{\prime}$ & Ar" & $\begin{array}{l}\text { Yield } \\
(\%)\end{array}$ & $\begin{array}{l}\text { M. P. } \\
\left({ }^{\circ} \mathrm{C}\right)\end{array}$ \\
\hline III a & C6H5 & Piperidine & C6H5 & C6H5 & 46 & $160-162$ \\
\hline III $b$ & C6H5 & Piperidine & C6H5 & 4-F-C6H4 & 48 & 173-174 \\
\hline III c & 4-Br-C6H4 & Piperidine & C6H5 & C6H5 & 47 & 163-164 \\
\hline III d & 4-Br-C6H4 & Piperidine & C6H5 & 4-F-C6H4 & 43 & 197-199 \\
\hline III e & 4-Br-C6H4 & Morpholine & C6H5 & 4-F-C6H4 & 42 & 172-174 \\
\hline III f & 4-Cl -C6H4 & Morpholine & C6H5 & C6H5 & 42 & $172-174$ \\
\hline III $g$ & 4-Cl-C6H4 & Morpholine & C6H5 & 4-F-C6H4 & 39 & 197-199 \\
\hline III $h$ & 4-Cl-C6H4 & Pyrolidine & C6H5 & C6H5 & 35 & $156-158$ \\
\hline III i & 4-Cl-C6H4 & Pyrolidine & C6H5 & $4-\mathrm{Br}-\mathrm{C} 6 \mathrm{H} 4$ & 38 & $137-138$ \\
\hline
\end{tabular}

Table:2 Antibacterial Activity of synthesized Compounds (III a-j)

\begin{tabular}{|l|l|l|l|l|l|l|}
\hline \multirow{2}{*}{ Comp. No. } & \multicolumn{4}{|l}{ MIC in $\mu \mathrm{g} / \mathrm{mL}$ (zone of inhibition, mm) } & \multicolumn{2}{l|}{ Antifungal activity } \\
\cline { 2 - 7 } & Antibacterial activity & S. aureus & E. coli & P. aeruginosa & A. niger & C. albicans \\
\cline { 2 - 7 } IIIa & $25(14)$ & $25(12)$ & $25(15)$ & $25(12)$ & $25(10)$ & $25(14)$ \\
\hline IIIb & $25(6)$ & $25(7)$ & $50(8)$ & $25(10)$ & $25(10)$ & $25(10)$ \\
\hline IIIc & $25(15)$ & $25(13)$ & $25(16)$ & $25(15)$ & $25(12)$ & $25(14)$ \\
\hline IIId & $50(12)$ & $50(10)$ & $25(14)$ & $25(13)$ & $50(14)$ & $50(12)$ \\
\hline IIIe & $25(10)$ & $25(10)$ & $50(14)$ & $50(12)$ & $50(10)$ & $50(10)$ \\
\hline IIIf & $25(15)$ & $50(12)$ & $25(17)$ & $50(14)$ & $25(10)$ & $25(10)$ \\
\hline IIIg & $25(10)$ & $100(10)$ & $25(12)$ & $25(12)$ & $50(10)$ & $50(10)$ \\
\hline IIIh & $50(10)$ & $50(12)$ & $25(13)$ & $25(12)$ & $25(12)$ & $25(12)$ \\
\hline IIIi & $25(12)$ & $25(14)$ & $25(13)$ & $25(15)$ & $25(18)$ & $25(17)$ \\
\hline Streptomycin & $25(18)$ & $25(16)$ & $25(20)$ & $25(18)$ & ND & ND \\
\hline Nystatin & ND & ND & ND & ND & $6.25(16)$ & $25(18)$ \\
\hline
\end{tabular}

\section{Conclusion:}

In summary, this work provides mild and efficient methodology for the synthesis of tetra substituted aminonicotinonitrile. Compound III a, III c, III i found to be potent antibacterial agents against Bacillus subtilis, Escherichia coli and Pseudomonas aeruginosa respectively, where as compound III c, III f and III i shows predominant activity against Aspergillus niger and Candida albicans fungal species. 


\section{Acknowledgments:}

The authors are sincerely thankful to Principal Dr. N. V. Kalyankar Yeshwant Mahavidhyalaya Nanded for providing laboratory facilities for this work.

\section{References}

[1] A. Elbert, B. Becker, J Harwing, and C. Erdelen, Pflanzenschutz Nachrichten, 44, 1991, 113.

[2] A. Elbert, H. Overbeck, K. Lwaya and S. Tsuboi, Proc. Br. Crop. Prot. Conf-pest Dis, 01, 1990, 21.

[3] M. P. P. Raj, J. T. Rao, Asian J. Chem., 25, 2003, 492.

[4] B. S. Holla, C. S. Prassana, B. Poojari, K. S. Rao, K. Shridhara and U. G. Bhatt, Indian J. Chem, 33B, 2004, 643.

[5] A. D. Kennedy, A. L. Summers, J. Het. Chem. 18, 1981, 409.

[6] A. B. Ivoanov, D. Z. Levin, V. K. Promonekoe and E. S. Zh. Org. Khim, 25, 1989, 629.

[7] Moffett, R. B.; Robert, A.; Skaletzky, L. L. J. Med. Chem, 10, 1971, 963.

[8] C. J. Wilkins, J. E. Douglas, Inorg. Chim. Acta. 3, 1969, 635.

[9] V. N. Kozhevnikov, D. N. Kozhevnikov, T. V. Nikitina, V. L. Rusinov, O. L. Chupakhin, M. Zabel, B. J. Konig, Org. Chem. 68, 2003, 2882.

[10] Hantzsch, A. Justus Liebiegs Ann Chem, 215, 1882, 1.

[11] Sprung, M. M. Chem Rev, 71, 1940, 2629.

[12] Petrenko-Kritschenko, P.; Zoneff, N. Ber, 39, 1906, 1358.

[13] (a) Krohnke, F.; Zecher, W. Angew Chem Int Ed Engl, 1, 1962, 626. (b) Krohnke, F. Synthesis, 1, 1976.

[14] (a) Baron, H.; Remfry, F. G. P.; Thorpe, J. F. J Chem Soc, 85, 1904, 1726. (b) Vogel, A. I. J Chem Soc , 1758, 1934.

[15] Jones, G. In: Comprehensive Heterocyclic Chemistry; Katritzky, A. R., Rees, C. W., Eds.; Pregamon: New York, NY Vol. 2, Part 2, 1984, 395.

[16] Alvarez-Insua, A. S.; Lora-Tamayo, M.; Soto, J. L. J Heterocycl Chem, 7, 1970, 1305.

[17] Joule, J. A.; Smith, G.; Mills, K. Heterocyclic Chemistry,3rd ed.; Chapman and Hill: London, 72-119, 1995.

[18] Saikai, P.; Prajapati, D.; Sandhu, J. S. Tetrahedron Lett, 44, 2003, 8725.

[19] Penieres, G.; Garcia, O.; Franco, K.; Hernardez, O.; Alvarez, C. Heterocycl Commun, 2, 1996, 359.

[20] Gordeev, M. F.; Patel, D. V.; Gordon, E. M. J Org Chem, 61, 1996, 924.

[21] Potts, K. T.; Cipullo, M. J.; Ralli, P.; Theodoridies, G. J Am Chem Soc, 103, 1981, 3585.

[22] Pratap, R.; Kumar, B.; Ram, V. J. Tetrahedron, 63, 2007, 10309. 